\title{
Boundary-Domain Integral Equation Systems to the Dirichlet and Neumann Problems for Compressible Stokes Equations with variable viscosity in $2 \mathrm{D}$
}

\author{
Tsegaye Ayele ${ }^{1}$, Mulugeta Dagnaw ${ }^{2}$, and Sergey Mikhailov ${ }^{3}$ \\ ${ }^{1}$ Addis Ababa University \\ ${ }^{2}$ Addis Ababa University College of Natural Sciences \\ ${ }^{3}$ Brunel University London
}

May 5, 2020

\begin{abstract}
In this paper, the Dirichlet and Neumann boundary value problems for the steady-state Stokes system of partial differential equations for a compressible viscous fluid with variable viscosity coefficient is considered in two-dimensional bounded domain. Using an appropriate parametrix, this problem is reduced to a system of direct segregated boundary-domain integral equations (BDIEs). The BDIEs in the two-dimensional case have special properties in comparison with the three dimension because of the logarithmic term in the parametrix for the associated partial differential equations. Consequently, we need to set conditions on the function spaces or on the domain to ensure the invertibility of corresponding parametrix-based hydrodaynamic single layer and hypersingular potentials and hence the unique solvability of BDIEs. Equivalence of the BDIE systems to the Dirichlet and Neumann BVPs and the invertibility of the corresponding boundary-domain integral operators in appropriate Sobolev spaces are shown.
\end{abstract}

\section{Hosted file}

D_N_BDIE systems for compressible stokes Equations.pdf available at https://authorea.com/ users/292921/articles/420848-boundary-domain-integral-equation-systems-to-the-dirichletand-neumann-problems-for-compressible-stokes-equations-with-variable-viscosity-in-2d 